\title{
Identificação de Experiências da Adoção de Learning Analytics no Ensino de Engenharia de Software
}

\author{
Marcelo Werneck Barbosa - PUC Minas
}

\begin{abstract}
Resumo. O número de cursos de graduação e especialização em Engenharia de Software tem crescido nos últimos anos no Brasil. Atualmente, as instituições já dispõem de dados sobre a execução destes cursos que poderiam ser utilizados na análise de sua condução e identificação de melhorias. Uma possibilidade de avaliar este grande volume de dados pode ser por meio de Learning Analytics. Entretanto, existem poucos trabalhos ainda nesta área. Este trabalho exploratório-descritivo tem o objetivo de identificar experiências do uso de Learning Analytics no ensino de Engenharia de Software e fomentar a discussão deste tema. Foram encontrados poucos trabalhos neste contexto, o que demonstra a carência de estudos de Learning Analytics nesta área.
\end{abstract}

Palavras-chave: Learning Analytics, Engenharia de Software, Revisão Sistemática.

\section{Identifying experiences of the adoption of Learning Analytics on Teaching Software Engineering}

\begin{abstract}
The number of under graduation and graduation courses on Software Engineering has increased in the past years in Brazil. Currently, universities already have data on the execution of such courses that could be analyzed and used for identification of improvements. One possibility to evaluate such great volume of data is through Learning Analytics. However, there are still few articles in this area. This descriptive and exploratory study is aimed at identifying experiences on the use of Learning Analytics with Software Engineering courses and augmenting the discussion of the theme. Few studies were found in this context, which demonstrates the lack of research on Learning Analytics in this field.
\end{abstract}

Keywords: Learning Analytics, Software Engineering, Systematic Review.

\section{Introdução}

A qualidade da educação em Engenharia de Software (ES) pode contribuir significativamente para a melhoria do estado da arte do desenvolvimento de software e auxiliar a solução de alguns problemas tradicionais e crises relacionadas com as práticas da indústria de software. Hoje, a educação e o treinamento para formar profissionais de software devem incluir não apenas conhecimentos básicos na área de computação, mas também o ensino de conceitos, processos e técnicas para definição, desenvolvimento e manutenção de software. As abordagens mais comuns para ensinar Engenharia de Software incluem aulas expositivas, aulas de laboratório, entre outras. Entretanto, abordagens alternativas podem ajudar os alunos a aprender de maneira mais efetiva, como por exemplo, a substituição de aulas expositivas por discussão de casos práticos, dinâmicas de grupo e o uso de jogos (Prikladnicki et al., 2009). Um dos grandes desafios enfrentados no ensino da Engenharia de Software atualmente é suprir a necessidade de 
uso de métodos de ensino que permitam tornar o processo de ensino-aprendizagem mais efetivo. A aceleração das inovações tecnológicas também tem representado um desafio para o ensino em ES (Wangenheim, Kochanski e Savi, 2009).

Atualmente, a disciplina de Engenharia de Software é ensinada nos cursos da área de computação no Brasil, sejam eles cursos cuja computação é uma atividade-meio (Sistemas de Informação e Licenciatura em Computação) ou cursos cuja computação é uma atividade-fim (Ciencia da Computação e Engenharia de Computação) (Soares, 2004) assim como em cursos de pós-graduação (Cunha et al., 2008) (Prikladnicki et al., 2009). Os alunos egressos da disciplina de Engenharia de Software devem demonstrar habilidades para gerenciar projetos de software, analisar, projetar, verificar, validar, implementar e manter sistemas de software (Soares, 2004).

Segundo consulta direta no banco de dados e-MEC do Ministério da Educação (MEC, 2015), há registrados no Brasil 22 cursos de graduação em Engenharia de Software, sendo 1 deles a distância. Segundo a mesma base de dados, há no país 91 cursos de especialização com "Engenharia de Software" no título, sendo 9 deles a distância. Os primeiros relatos de cursos de graduação em Engenharia de Software no Brasil datam de 2010 (Figueiredo et al., 2010). Já os cursos de especialização neste tema existem há mais de uma década. Sendo assim, trata-se de uma área de ensino relativamente nova no país, mas que já possui um conjunto de dados da execução destes cursos que pode ser analisado e usado para propor melhorias para o aprendizado dos alunos. Uma das possibilidades para análise destes dados é por meio do uso de Learning Analytics.

Learning Data Analysis (LA, Learning Analytics) é um campo de pesquisa relativamente novo (Agudo-Peregrina et al., 2012). O conceito de Learning Analytics tem atraído atenção significativa de diversas comunidades com interesses na intersecção entre aprendizagem e tecnologia da informação, incluindo administradores da área de educação, serviços computacionais corporativos, educadores e estudantes (Shu e Ferguson, 2012). Learning Analytics tem o propósito de identificar padrões de comportamento e tendências na aprendizagem a partir de conjuntos massivos de dados relacionados à educação para promover o desenvolvimento de uma educação mais personalizada (Zhu et al., 2014). Com a disponibilidade destas grandes quantidades de dados sem precedentes sobre atividades e interesses dos alunos, há um potencial significativo de se fazer melhor uso dos mesmos para melhorar os resultados da aprendizagem (Shu e Ferguson, 2012). Esta grande quantidade de dados, também chamada de big data, pode estar registrada nas bases de dados de ambientes virtuais de aprendizagem. Este volume é considerado grande demais para se realizar uma análise usando ferramentas de base de dados tradicionais e este fato torna necessário o desenvolvimento de ferramentas e filtragem de dados específicos para se extrair informação útil destes dados (Agudo-Peregrina et al., 2012).

Neste contexto, este artigo tem o propósito de identificar experiências realizadas com a aplicação de Learning Analytics no ensino de Engenharia de Software. Por se tratar de uma área nova, espera-se que os resultados encontrados possam ser usados como direção inicial para pesquisadores brasileiros da área e que também seja fomentada a discussão sobre o tema.

O restante deste artigo está organizado da seguinte forma. A Seção 2 apresenta o referencial teórico sobre Learning Analytics. A Seção 3 descreve a metodologia utilizada neste trabalho. A Seção 4 apresenta os relatos encontrados sobre o uso de Learning 
Analytics no ensino de Engenharia de Software enquanto a Seção 5 discorre sobre as conclusões do artigo e possíveis trabalhos futuros.

\section{Learning Analytics}

Learning Analytics é a terceira onda de desenvolvimento na tecnologia instrucional, que iniciou com o advento dos sistemas de gerenciamento de aprendizagem em 1991. A segunda onda integrou estes sistemas a uma organização mais ampla envolvendo os alunos em redes sociais (também conhecida como onda Web 2.0) (Fiaidhi, 2014). Learning Analytics tem suas raízes em duas iniciativas computacionais não especificamente preocupadas com a aprendizagem, mas sim com fortes demandas de negócio para compreender dados internos das organizações e comportamento do consumidor e cliente externos: Business Intelligence e Data Mining. Do campo de Data Mining, se desenvolveu o campo de Educational Data Mining (EDM). Os projetos de pesquisa se multiplicaram após o amplo uso de Ambientes Virtuais de Aprendizagem (AVA) no início do século XXI (Shu e Ferguson, 2012). A proliferação destes ambientes virtuais em todos os setores da educação produz vastas quantidades de dados que podem ser rastreados (Greller e Drachsler, 2012). Estes ambientes consistem em mídias que utilizam o ciberespaço para veicular conteúdos e permitir interação entre os atores do processo educativo. Qualquer ambiente virtual de aprendizagem deve permitir diferentes estratégias de aprendizagem, não só para se adequar ao maior número possível de pessoas, que terão certamente estratégias diferenciadas, mas também porque as estratégias utilizadas individualmente variam de acordo com fatores como interesse, familiaridade com o conteúdo, estrutura dos conteúdos, motivação e criatividade, entre outros. Além disso, deve proporcionar a aprendizagem colaborativa, interação e autonomia (Messa, 2010).

A premissa de Learning Analytics é a coleção e pré-tratamento de dados sobre comportamento dos usuários. Estes dados são coletados a partir de ações explícitas dos alunos, tais como completar tarefas, realizar exames, interações online, realização de atividades e participação em fóruns de discussão, e outras atividades que não são diretamente avaliadas como parte do progresso educacional do aluno, como emoção e personalidade (Zhu et al., 2014). LA envolve o processamento destes dados e pode ser definida como a medição, coleta, análise e reporte de dados sobre alunos e seus contextos, com o propósito de compreender e otimizar a aprendizagem e os ambientes nos quais ela ocorre (Ferguson, 2012). LA tem como objetivos: apoiar a elaboração de currículos, prever o desempenho de alunos, melhorar o ambiente de ensinoaprendizagem, apoiar tomadas de decisão para instituições e cursos e desenvolver abordagens mais individualizadas para os alunos (Suchithra, 2015). LA e data mining educacional estão em seus passos iniciais e ferramentas estão surgindo lentamente (del Blanco et al., 2013).

\section{Procedimentos Metodológicos}

Este estudo é classificado como exploratório-descritivo, pois o seu objetivo é ampliar o conhecimento a respeito de um determinado fenômeno, explorar a realidade e buscar maior conhecimento sobre um tema para depois detalhar os fatos descobertos. A abordagem adotada foi a qualitativa, que se concentra no aprofundamento dos valores contidos nos dados. Esse tipo de análise tem por base conhecimentos teóricos e empíricos que permitem atribuir-lhe cientificidade (Zanella, 2009). 
O trabalho se iniciou com a formulação de uma pergunta de pesquisa "Que trabalhos têm sido desenvolvidos com a aplicação de Learning Analytics no contexto de ensino de Engenharia de Software? Para responder a esta pergunta, foi adotado um procedimento similar à realização de uma revisão sistemática com base no processo definido por Kitchenham (2004). A revisão sistemática é uma ferramenta utilizada para identificar, avaliar e interpretar toda pesquisa disponível e relevante sobre uma questão de pesquisa, um tópico ou um fenômeno de interesse, ou seja, uma forma de executar revisões abrangentes da literatura de forma não tendenciosa. Esse tipo de investigação disponibiliza um resumo das evidências relacionadas a uma estratégia de intervenção específica mediante a aplicação de métodos explícitos e sistematizados de busca, apreciação crítica e síntese da informação selecionada. A revisão sistemática é útil para integrar as informações do conjunto de estudos realizados separadamente sobre determinada visão, que podem apresentar resultados conflitantes e/ou coincidentes, bem como evidenciar temas e auxiliar na orientação das intervenções (Sampaio e Mancini, 2007).

Por se tratar de uma área nova de pesquisa, poucos artigos foram encontrados relacionando os dois temas. Buscou-se por artigos em inglês e português, disponíveis em quatro bases de dados eletrônicas: IEEEXplore, ACM Digital Library, EBSCO e Portal Capes de Periódicos. Para obtenção das publicações foram utilizadas nestas bases pesquisas pelas palavras-chave: "learning analytics" AND "software engineering" e ainda "learning analytics" AND "engenharia de software".

Os artigos encontrados pelo procedimento de revisão sistemática que aparentemente estavam relacionados ao objetivo de pesquisa foram coletados. Por serem poucos os artigos retornados, foi realizada uma leitura completa dos mesmos e sua análise está apresentada na Seção 4 deste trabalho. Após leitura e análise dos trabalhos encontrados, foram buscados trabalhos relacionados ao tema de pesquisa na seção de referências de cada um dos artigos, mas não foram encontrados outros trabalhos que unissem tanto Learning Analytics quanto Engenharia de Software.

\section{Experiências do uso de Learning Analytics no Ensino de Engenharia de Software}

Nesta seção, são apresentados os trabalhos que tratam do uso de Learning Analytics no ensino de Engenharia de Software encontrados pelo procedimento descrito anteriormente. O passo final de uma revisão sistemática é a apresentação dos resultados. Uma forma de síntese da pesquisa é uma revisão narrativa que procura identificar o que tem sido escrito e publicado sobre um determinado assunto. Esta narrativa á apresentada nesta seção.

(Conde et al., 2014) apresentaram um sistema visual de Learning Analytics que torna possível a exploração de informações sobre a interação entre alunos em plataformas de aprendizagem. $\mathrm{O}$ sistema inclui diversas ferramentas que auxiliam a análise da interação de usuários em algumas dimensões como: quando a interação foi realizada, quais conteúdos são mais importantes para os usuários, como eles interagem entre si, e outros. $\mathrm{O}$ sistema foi testado com informações registradas durante 5 anos acadêmicos. O sistema conseguiu identificar momentos dos cursos com menor participação dos alunos com o objetivo de propor novas atividades para homogeneizar sua participação ao longo do curso. A ferramenta possibilitou ainda identificar os conteúdos mais relevantes por meio da análise dos fóruns de discussão. Desta maneira, 
foi possível incluir mais tópicos, ferramentas e atividades de acordo com o interesse dos alunos. O sistema também teve o propósito de identificar os alunos com maior participação e como eles interagem com os recursos da ferramenta de modo a definir estratégias para aumentar a participação de alunos e professores. Por fim, o trabalho conseguiu observar ainda que algumas atividades têm impactos positivos ou negativos nas notas dos alunos. Desta forma, algumas atividades podem ser promovidas ou evitadas.

(Pratheesh e Devi, 2013a) analisaram, por meio de Learning Analytics, a influência de estilos de aprendizagem na aquisição de conhecimento com a aplicação de 46 questionários aplicados a alunos de um curso de MBA em aplicações computacionais. Os autores defendem que o estilo de aprendizagem tem um papel vital na aquisição de conhecimento, o que também é aplicável à Engenharia de Software. Segundo eles, o uso de Learning Analytics auxilia a medir e melhorar as atividades de aprendizagem de Engenharia de Software e a produzir conhecimentos nos alunos que irão satisfazer as necessidades da indústria. O trabalho identificou que a maioria dos alunos em uma disciplina de Engenharia de Software não possui apenas um estilo de aprendizagem e prefere a adoção de múltiplos estilos ao estudar este assunto. Os autores concluem que muitas disciplinas deste tema ainda praticam métodos tradicionais de ensino e que os alunos sentem dificuldades em lidar com problemas do mundo real de maneira apropriada.

Como continuação do trabalho descrito anteriormente, Pratheesh e Devi (2013b) propuseram um sistema web de aprendizagem colaborativa que dinamicamente identifica o estilo de aprendizagem do aluno e sugere materiais de estudo online compatíveis com seu estilo. O sistema conta com funcionalidades como materiais de aprendizagem por blogs, módulo de avaliação individual online, atividades por chat entre outras. Os autores identificaram que alunos de cursos de Engenharia de Software possuem todos os estilos de aprendizagem como visuais, auditivos e sinestésicos. A maioria dos alunos de ES prefere ambientes colaborativos de aprendizagem com uso de tecnologia em vez de métodos tradicionais.

O artigo de Pratheesh e Devi (2015), encontrado pelo processo de revisão, é uma continuação dos trabalhos anteriores. Os autores avaliaram técnicas de ensino de Engenharia de Software como projetos em grupo, jogos educacionais e aprendizado pela web. Foram avaliados construtos relacionados ao engajamento dos alunos no curso. Os resultados da pesquisa mostram que os estudantes não têm uma postura ativa no ensino tradicional; consideram este método entediante e não investem tempo em seu aprendizado com ele. Os alunos preferem assistir a vídeos sobre Engenharia de Software, buscar informações na internet, participar de chats com colegas e fóruns de discussão. Os alunos esperam que os professores os encorajem a contrapor opiniões diferentes e obter conhecimento por meio de fóruns de discussões em vez de por meio de memorização.

Mittal e Sureka (2014) apresentaram uma abordagem para coletar dados de repositórios de dados gerados a partir da construção de software por grupos de alunos em um curso de Engenharia de Software. Três tipos de repositórios foram analisados: wiki (para etapa de engenharia de requisitos), sistema de controle de versão (para desenvolvimento e manutenção do código) e sistema de acompanhamento de itens (para manutenção adaptativa e corretiva). Foram propostas métricas, visualizações e algoritmos usados para analisar e interpretar os dados dos trabalhos dos alunos com o 
propósito de fornecer feedback para professores e aumentar a qualidade dos trabalhos dos alunos. Foram analisados dados de 19 grupos com 5 membros cada. Ao observar padrões de commit, os autores perceberam que havia grupos com distribuição da carga de trabalho desigual, maior intensidade de trabalho próximo ao prazo final de entrega e também qualidade na resolução de defeitos e aderência aos processos definidos.

Não foram encontrados trabalhos realizados por pesquisadores brasileiros nesta área, o que mostra o potencial de estudos a serem desenvolvidos neste contexto em nosso país. Dos 5 trabalhos encontrados, 4 foram encontrados por meio de pesquisas no portal IEEExplore e apenas 1 encontrado na base da ACM Digital Library. Pelo Portal da CAPES não foi encontrado nenhum trabalho por meio das palavras-chave informadas. A base de trabalhos científicos EBSCO também foi utilizada, mas as pesquisas usando as palavras-chave só retornaram trabalhos já encontrados nas outras bases.

Pode-se observar também que todos os artigos encontrados são bastante recentes, sendo que 2 foram publicados no ano de 2013, 2 em 2014 e 1 no ano de 2015. Outro dado interessante é que 4 artigos foram publicados em meios específicos da área de Computação e apenas 1 foi publicado em evento relacionado à área de Educação. A Tabela 1 resume os dados dos trabalhos encontrados pelo procedimento de revisão sistemática.

Tabela 1. Artigos encontrados pela revisão sistemática

\begin{tabular}{|c|c|c|c|}
\hline Referência & Título & Evento & Base de dados \\
\hline Conde et al. (2014) & $\begin{array}{l}\text { Visual learning } \\
\text { analytics techniques } \\
\text { applied in software } \\
\text { engineering subjects }\end{array}$ & $\begin{array}{l}\text { Frontiers in Education } \\
\text { Conference }\end{array}$ & IEEExplore \\
\hline $\begin{array}{l}\text { Pratheesh e Devi } \\
\text { (2013a) }\end{array}$ & $\begin{array}{l}\text { Influence of Learning } \\
\text { Analytics in Software } \\
\text { Engineering } \\
\text { Education }\end{array}$ & $\begin{array}{l}\text { International } \\
\text { Conference on } \\
\text { Emerging Trends in } \\
\text { Computing, } \\
\text { Communication and } \\
\text { Nanotechnology }\end{array}$ & IEEExplore \\
\hline $\begin{array}{l}\text { Pratheesh e Devi } \\
\text { (2013b) }\end{array}$ & $\begin{array}{l}\text { Sensation of Learning } \\
\text { Analytics to Prevail } \\
\text { the } \\
\text { Engineering } \\
\text { Education }\end{array}$ & $\begin{array}{l}\text { International } \\
\text { Conference on } \\
\text { Advanced Computing } \\
\text { and Communication } \\
\text { Systems }\end{array}$ & IEEExplore \\
\hline $\begin{array}{l}\text { Pratheesh e Devi } \\
\text { (2015) }\end{array}$ & $\begin{array}{l}\text { Necessity of Learning } \\
\text { Analytics in Software } \\
\text { Engineering } \\
\text { Education }\end{array}$ & $\begin{array}{l}\text { Journal of } \\
\text { Engineering Science } \\
\text { and Technology }\end{array}$ & IEEExplore \\
\hline $\begin{array}{l}\text { Mittal e Sureka } \\
(2014)\end{array}$ & $\begin{array}{l}\text { Process } r \text { mining } \\
\text { software repositories } \\
\text { from student projects } \\
\text { in an undergraduate } \\
\text { software engineering } \\
\text { course }\end{array}$ & $\begin{array}{l}\text { International } \\
\text { Conference } \\
\text { Software } \\
\text { Engineering }\end{array}$ & ACM Digital Library \\
\hline
\end{tabular}


Como limitações do trabalho, pode-se citar o fato de terem sido usadas poucas palavras-chave, o que de certa forma, limita a busca e pode impedir que outros trabalhos sejam encontrados. Entretanto, as palavras-chave foram limitadas desta maneira para atender ao objetivo da pesquisa, pois os trabalhos analisados precisam tratar simultaneamente dos dois temas: Engenharia de Software e Learning Analytics.

Neste mesmo contexto, por ser uma área de estudo ainda em seus passos iniciais e com ferramentas surgindo lentamente, conforme afirma del Blanco et al. (2013), não foi possível caracterizar o conceito de Learning Analytics de maneira mais específica a ponto de se encontrar outras palavras-chave que pudessem ser utilizadas na busca. Entende-se que é possível que haja palavras que representem técnicas, processos ou ferramentas relacionados a Learning Analytics que tenham sido usadas em trabalhos relacionados a Engenharia de Software, sem menção específica ao termo Learning Analytics. Neste caso, estes trabalhos não terão sido encontrados realmente pela busca realizada.

\section{Conclusões e trabalhos futuros}

Há uma vasta quantidade de dados de instituições de ensino à disposição para auxiliar os administradores destas instituições a atingir seus objetivos de melhorar o desempenho dos estudantes e aumentar a retenção dos mesmos. Alguns destes dados que podem ser analisados são: número de vezes que um recurso foi acessado, notas, data e número de discussões e postagens em fóruns online e atividades realizadas (Dietz-Uhler \& Hurn, 2013). Blackboard e Moodle são exemplos bem conhecidos de ambientes virtuais de ensino. São ferramentas que armazenam grande quantidade de dados relacionados às atividades dos alunos e seu perfil pessoal (Shum e Ferguson, 2012). Com o número crescente de cursos presenciais e a distância de Engenharia de Software no país, uma quantidade maior de dados sobre os alunos e seu desempenho pode ser analisada por meio de LA com o objetivo de melhorar os cursos e aumentar o aprendizado e interesse dos alunos.

O uso de Learning Analytics neste contexto permite a adoção de ferramentas e técnicas para analisar estes dados e uso dos mesmos na melhoria dos cursos. Este artigo teve o propósito de identificar experiências realizadas com a aplicação de Learning Analytics no ensino de Engenharia de Software e espera como contribuição iniciar a discussão de LA neste contexto no Brasil. Foram encontrados apenas 5 trabalhos nas bases consultadas envolvendo direta e explicitamente o uso de LA neste contexto. A adoção de LA não é tão recente mas, apesar de existirem já conferências específicas sobre o tema, seu uso no ensino de Engenharia de Software ainda é bastante incipiente no mundo e parece não ter iniciado no Brasil.

Dawson et al. (2014) analisaram as tendências de publicações na principal conferência e periódico sobre LA e identificaram que existe uma fragmentação nas disciplinas principais, ciência da computação e educação, em relação à representação nestes dois meios. Há ainda uma diversidade dos métodos usados pelos autores, mas os autores oriundos da área de educação tendem a usar métodos mais rigorosos. Autores da área de Ciência da Computação tendem a escrever relatórios, relatos de experiências pessoais e estatísticas aleatórias obtidas com tais experiências. Tal resultado também aponta a importância de métodos mais rigorosos em trabalhos que envolvam a área de Ciência da Computação e consequentemente Engenharia de Software. 
O principal evento sobre ensino em Engenharia de Software no Brasil é o Fórum em Educação de Engenharia de Software (FEES), realizado dentro do Congresso Brasileiro de Software (CBSoft). Na chamada de trabalhos da edição deste ano, o tema Learning Analytics sequer aparece na lista de tópicos de interesse do evento. Historicamente, o tipo de trabalho publicado neste fórum corrobora os resultados encontrados por Dawson et al. (2014), pois os trabalhos tratam, em sua maioria, de relatos de experiência isolados e dados obtidos com estas experiências.

Como trabalhos futuros, espera-se estudar a adoção de ferramentas de Learning Analytics, já empregadas em outros contextos, no ensino de Engenharia de Software. Pretende-se também avaliar seu uso em disciplinas com histórico de alto nível de reprovação em cursos de graduação relacionados a Informática, como disciplinas de ensino de programação de computadores.

Espera-se ainda que, com o crescimento dos cursos de Engenharia de Software no país, mais dados sejam coletados de forma padronizada e por mais tempo e que as experiências adotadas sejam mais contínuas de modo a possibilitar uma análise mais profunda dos resultados das mesmas. Assim, técnicas e ferramentas de Learning Analytics poderiam contribuir para o desenvolvimento e melhoria do campo.

\section{Referências}

Agudo-Peregrina, A. F.; Hernández-García, A.; Iglesias-Pradas, S. Predicting academic performance with learning analytics in virtual learning environments: A comparative study of three interaction classifications. In: International Symposium on Computers in Education, Andorra la Vella, 2012, pp. 1-6.

del Blanco, A.; Serrano, A.; Freire, M.; Martínez-Ortiz, I.; Fernández-Manjón, B. ELearning Standards and Learning Analytics Can Data Collection Be Improved by Using Standard Data Models? In: IEEE Global Engineering Education Conference (EDUCON), Berlin, 2013, pp 1255 - 1261.

Conde, M. A.; García-Peñalvo, F. J.; Gómez-Aguilar, D. A.; Theron, R. Visual learning analytics techniques applied in software engineering subjects. In: Frontiers in Education Conference (FIE), Madrid, 2014, pp.1-9.

Cunha, A. M.; Braga e Silva, G.; Monte-Mor, J. A.; Domiciano, M. A. P.; Vieira, R. G. Estudo de Caso Abrangendo o Ensino Interdisciplinar de engenharia de Software. In: Fórum de Educação em Engenharia de Software, 2008.

Dawson, S.; Gasevic, D.; Siemens, G.; Joksimovic, S. Current State and Future Trends: A Citation Network Analysis of the Learning Analytics Field. In: Learning Analytics and Knowledge (LAK), 2014, pp.231-240.

Dietz-Uhler, B.; Hurn, J. E. Using Learning Analytics to Predict (and Improve) Student Success: A Faculty Perspective. Journal of Interactive Online Learning, Volume 12, Number 1, 2013.

Ferguson, R. The State Of Learning Analytics in 2012: A Review and Future Challenges. Technical Report KMI-12-01, Knowledge Media Institute, The Open University, UK, 2012 .

Fiaidhi, J. The Next Step for Learning Analytics. IT Pro, September/October 2014. 
Figueiredo, R. M. C.; Ribeiro, L. C. M.; Sales, A. B.; Canedo, E. D.; Chaim, R. M.; Rocha, A.; Santos, A. R.; Ramos, C. S. Graduação em Engenharia de Software: uma proposta de flexibilização e interdisciplinaridade. In: III Fórum de Educação em Engenharia de Software (FEES), 2010.

Greller, W.; Drachsler, H. Translating Learning into Numbers: A Generic Framework for Learning Analytics. Educational Technology \& Society, 15 (3), pp. 42-57, 2012.

Kitchenham, B. A. Procedures for Performing Systematic Reviews. Tech. report TR/SE0401, Keele University, 2004.

MEC. Instituições de Educação Superior e Cursos Cadastrados. Disponível em $<$ http://emec.mec.gov.br/emec >. Acesso em 10 de julho de 2015.

Messa, W. C. Utilização de Ambientes Virtuais de Aprendizagem - AVAS: A Busca por uma Aprendizagem Significativa. Revista Brasileira de Aprendizagem Aberta e a Distância, Vol 9, 2010.

Mittal, M.; Sureka, A. Process mining software repositories from student projects in an undergraduate software engineering course. In: International Conference on Software Engineering (ICSE), pp. 344-353, 2014.

Pratheesh, N.; Devi, T. Influence of Learning Analytics in Software Engineering Education. In: International Conference on Emerging Trends in Computing, Communication and Nanotechnology (ICECCN 2013), 2013a.

Pratheesh, N.; Devi, T. Sensation of Learning Analytics to Prevail the Software Engineering Education. In: International Conference on Advanced Computing and Communication Systems (ICACCS -2013), 2013b.

Pratheesh, N.; Devi, T. Necessity of Learning Analytics in Software Engineering Education. Journal of Engineering Science and Technology Vol. 10, No.3, pp. 269 $-281,2015$.

Prikladnicki, R., Albuquerque, A. B., Wangenheim, C. G., Cabral, R. Ensino de Engenharia de Software: Desafios, Estratégias de Ensino e Lições Aprendidas. In: Fórum de Educação em Engenharia de Software (FEES), 2009.

Sampaio, R. F.; Mancini, M. C. (2007). Estudos de revisão sistemática: um guia para síntese criteriosa da evidência científica. Revista Brasileira de Fisioterapia, 11(1), 83-89.

Suchithra R.; Vaidhehi, V.; Iyer, N. E. Survey of Learning Analytics based on Purpose and Techniques for Improving Student Performance. International Journal of Computer Applications, Volume 111, No 1, Fevereiro de 2015.

Shum, S. B.; Ferguson, R. Social Learning Analytics. Educational Technology \& Society, 15 (3), 3-26, 2012.

Soares, M. S. Uma Experiência de Ensino de Engenharia de Software Orientada a Trabalhos Práticos. In: XXIV Congresso do SBC, XII WEI - Workshop de Educação em Computação, 2004.

Wangenheim, C. G.; Kochanski, D.; Savi, R. Revisão Sistemática sobre Avaliação de Jogos Voltados para aprendizagem de Engenharia de Software no Brasil. In: Fórum 
de Ensino de Engenharia de Software (FEES), Simpósio Brasileiro de Engenharia de Software (SBES), 2009.

Zanella. L. C. H. Metodologia de estudo e de pesquisa em administração. Florianópolis: Departamento de Ciências da Administração/UFSC, 2009.

Zhu, H.; Zhang, X.; Wang, X.; Chen, Y.; Zeng, B. A case study of learning action and emotion from a perspective of learning analytics. In: International Conference on Computational Science and Engineering, 2014. 